\title{
A possible LHCb Luminosity Monitor based on the Muon System
}

Sofia Kotriakhova
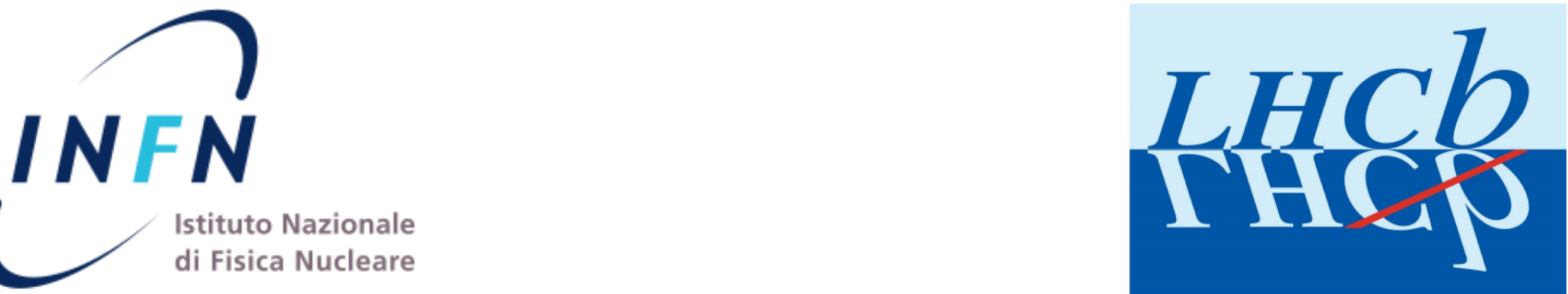


\section{LHCb experiment: the past}

The $\mathrm{LHCb}$ detector is a single-arm forward spectrometer covering the pseudorapidity range $1.9<\eta<4.9$, designed for the study of particles containing $b$ or $c$ quarks.

Trigger system: LO hardware trigger \& High Level Trigger (HLT)

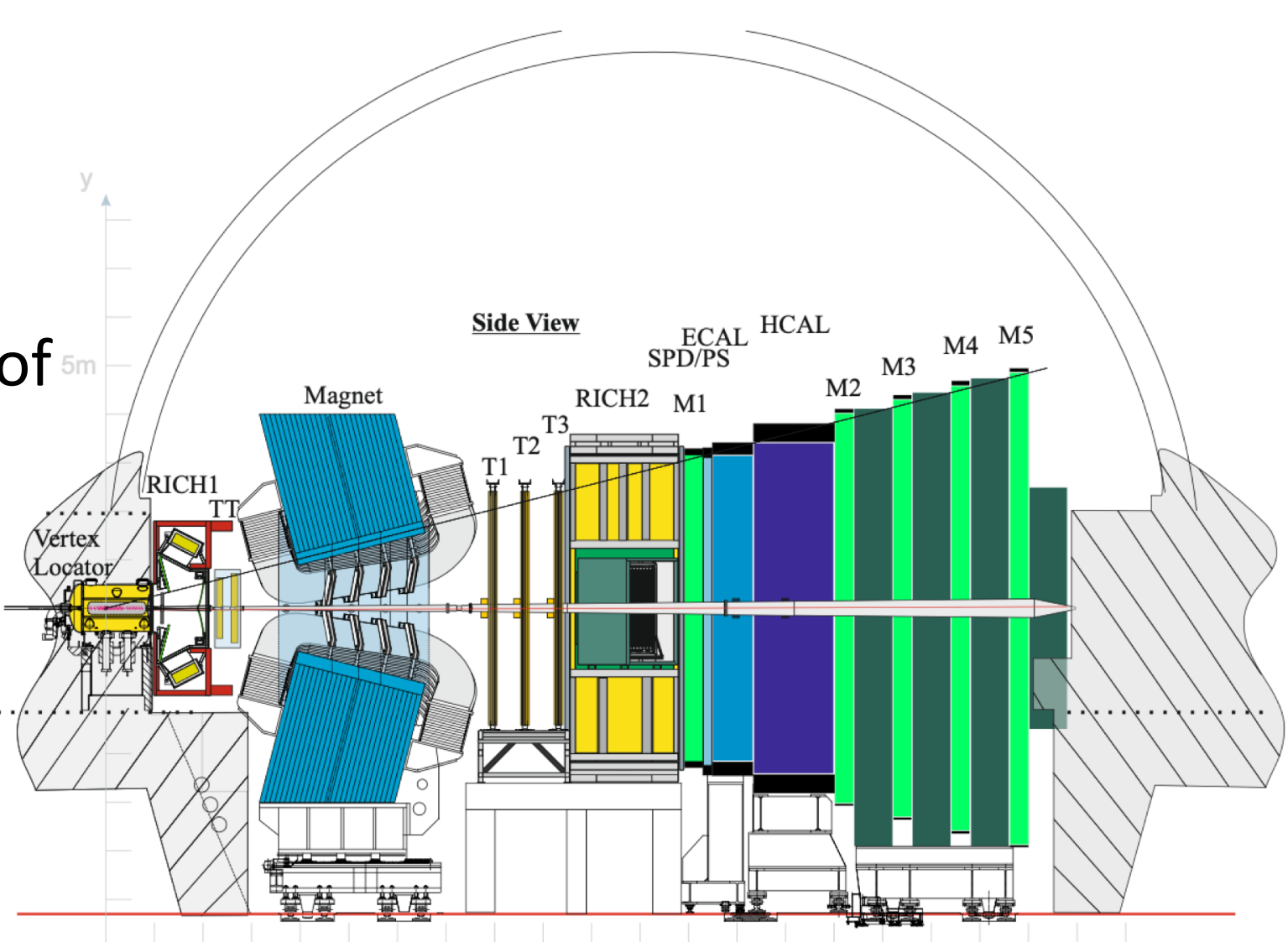




\section{LHCb experiment: the future}

The most sensitive part upgraded:

- New vertex locator

- New silicon strip detector

- New scintillating fibre detector

- New Front-End electronics

- No hardware trigger

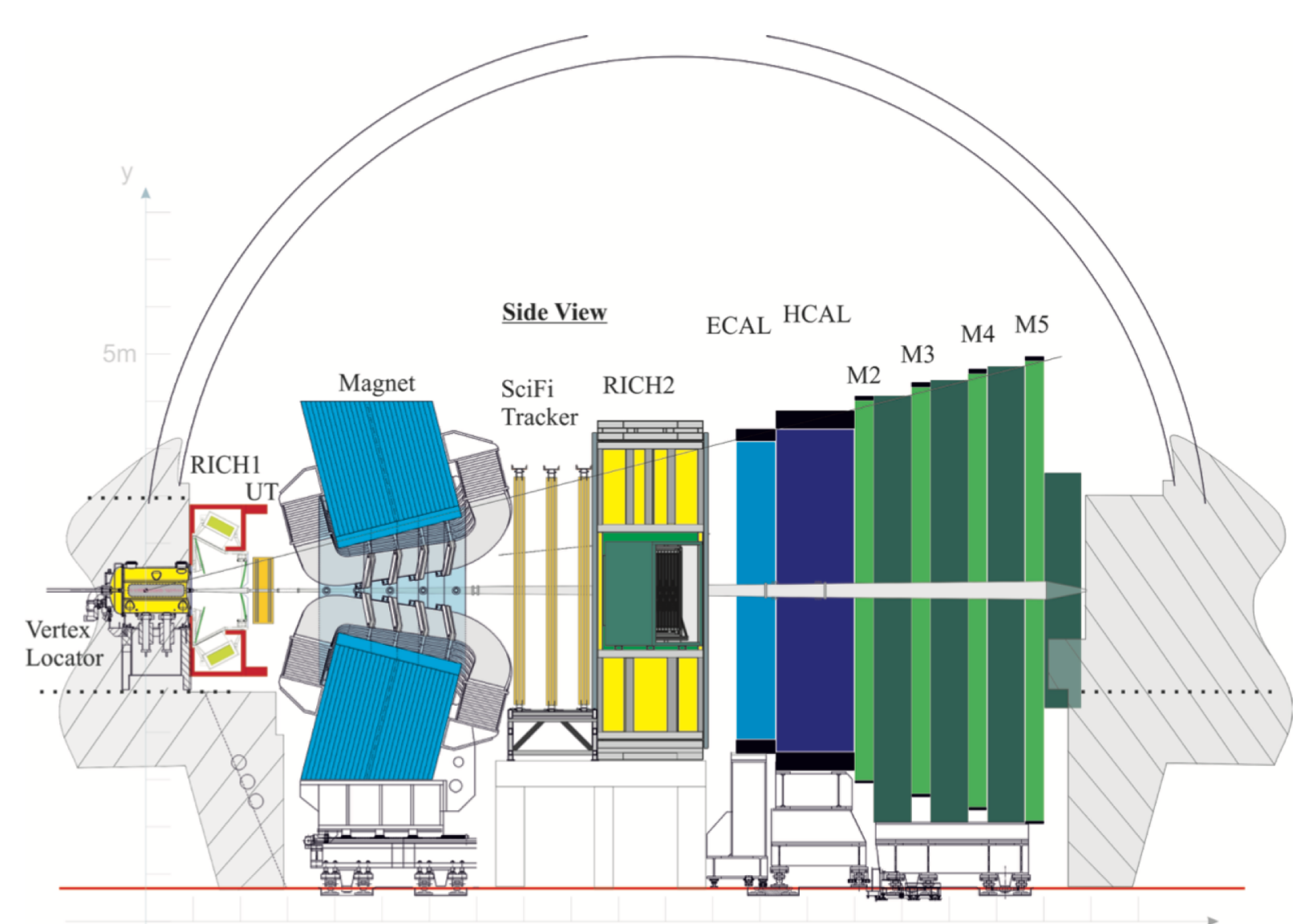




\section{Muon front-end electronics}

- CARDIAC readout board has 16 free-running scalers with preselectable gate

- The dead-time of the scalers is negligible compared with that of the CARDIAC

- Electronics deadtime depends on several parameters: $C_{D E T}$, signal shape (i.e. ionization, gas gain...), and was estimated from direct measurements of particle rates 


\section{Rates of muon system}

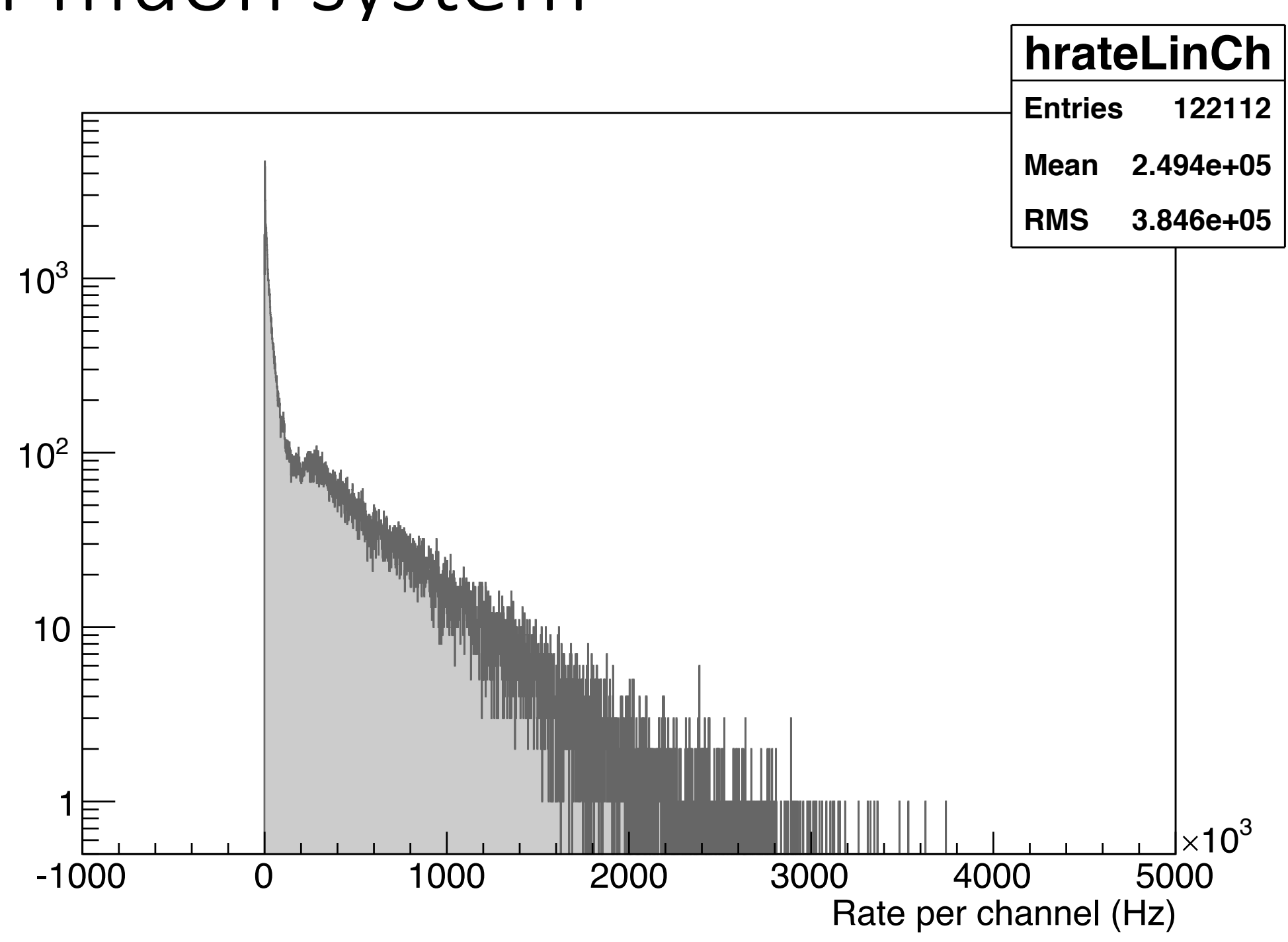




\section{Correction of the deadtime inefficiency}

The counting rate $R^{*}$ of readout channel is: $\quad R^{*}=R_{\text {part }}\left(1-\delta_{c} R^{*}\right)$

where $\delta_{c}$ is the CARIOCA dead time and $R_{\text {part }}$ is the rate of hitting particles.

The value of $\delta_{c}$ can be deduced from two measurements $\left(R_{i}^{*}\right.$ and $\left.R_{j}^{*}\right)$ performed at two different luminosities $\left(L_{i}\right.$ and $\left.L_{j}\right)$ :

$$
\left\{\begin{array}{l}
R_{i}^{*}=R_{\mathrm{part}}^{(i)}\left(1-\delta_{c} R_{i}^{*}\right) \\
R_{j}^{*}=R_{\mathrm{part}}^{(j)}\left(1-\delta_{c} R_{j}^{*}\right)
\end{array}\right.
$$

For each readout channel the ratio $\rho_{i j}$, which can be evaluated from the experimental data

$$
\rho_{i j}=\frac{R_{j}^{*} / L_{j}}{R_{i}^{*} / L_{i}}
$$

We get $\quad \rho_{i j}=1-\delta_{c}\left(1-\beta_{i j}\right) R_{j}^{*}$

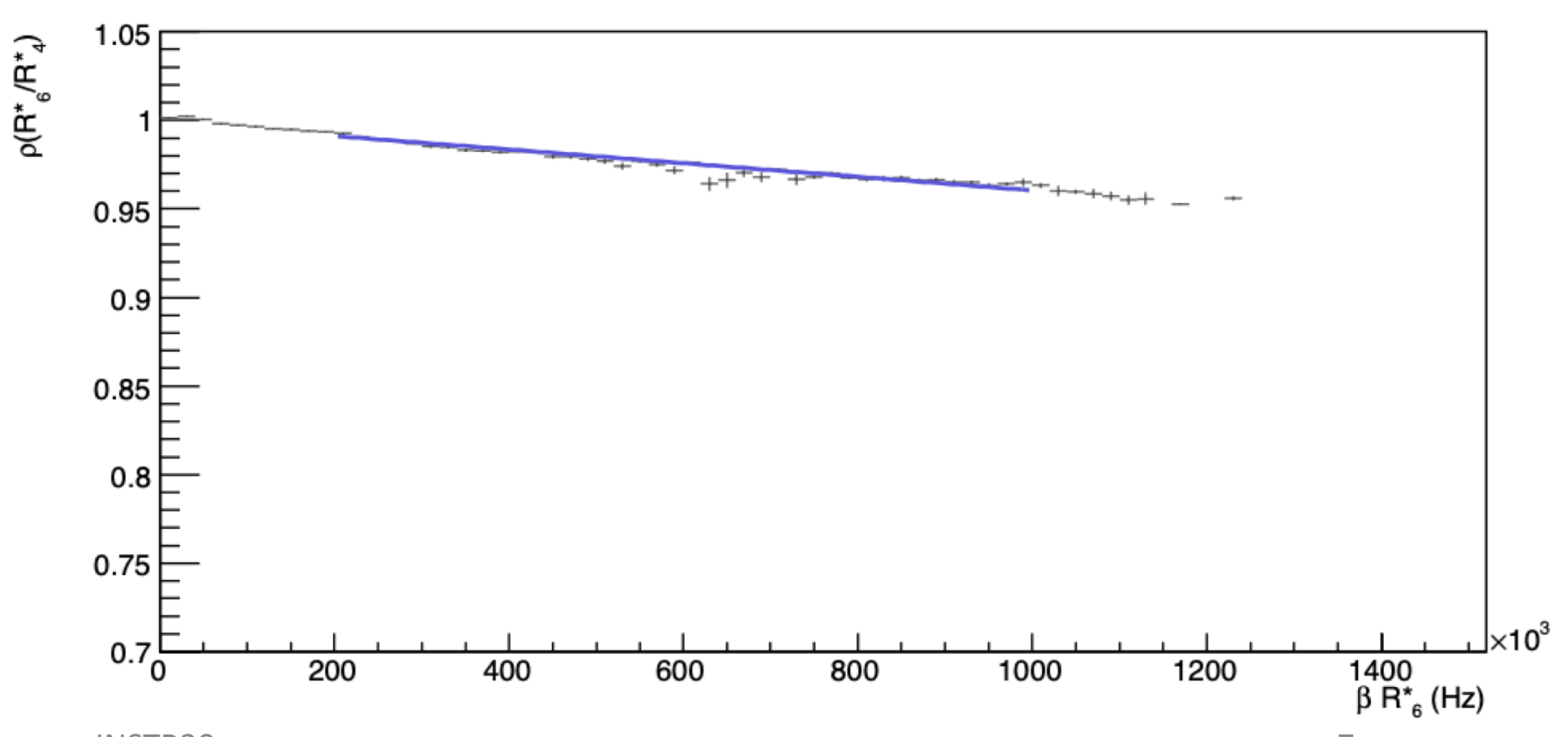

24.02.2020 $\quad\left(\beta_{i j}=L_{i} / L_{j}<1\right)$ 


\section{Correction of the deadtime inefficiency}

The detailed explanation of the deadtime correction method and results are given in dedicated paper

$$
\text { Jinst }
$$

Measurement of the front-end dead-time of the LHCb muon detector and evaluation of its contribution to the muon detection inefficiency

L. Anderlini, ${ }^{a}$ M. Anelli, ${ }^{b}$ F. Archilli, ${ }^{c}$ G. Auriemma, ${ }^{d, e}$ W. Baldini $, c, f$ G. Bencivenni, ${ }^{b}$ A. Bizzeti, ${ }^{a, g}$ V. Bocci, ${ }^{d}$ N. Bondar, ${ }^{c h}$ W. Bonivento, ${ }^{i}$ B. Bochin, ${ }^{h}$ C. Bozzi, ${ }^{c, f}$ D. Brundu, ${ }^{2}$ S. Cadeddu, 'P. Campana, ${ }^{\prime}$ G. Carboni, ${ }^{\prime, h}$ A. Cardini, ${ }^{\prime}$ M. Carletti, ${ }^{,}$L. Casu, A. Chubykin, ${ }^{h}$ P. Ciambrone, ${ }^{b}$ E. Dane, ${ }^{b}$ P. De Simone, ${ }^{b}$ A. Falabella, ${ }^{2}$ G. Felici, ${ }^{b}$ M. Fiore, ${ }^{c, f, m}$ M. Fontana,

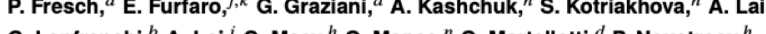
G. Lantranchi, A. Lol, O. Maev," G. Manca," G. Martellou," P. Neustroev," R.G.c. Oldeman," M. Palutan," G. Passaleva, G. Penso, D. Pinci, E. Polycarpo, ${ }^{9}$ B. Saitta, ${ }^{,}$R. Santacesaria, ${ }^{d}$ M. Santimaria, ${ }^{b}$ E. Santovetti, B.G. Siddi, ${ }^{f}$ G. Tellarini, ${ }^{f, m}$ C. Vacca, ${ }^{c, i}$ R. Vazquez-Gomez, ${ }^{b}$ S. Vecchi, ${ }^{\prime}$ M. Veltri ${ }^{a, r}$ and A. Voridi, ${ }^{h}$

A Secione INFN di Firenze, Firenze, Ittory

cati, Frascati, Italy

ear Research (CERN), Geneva, Switzerland "Sezione INFN di Roma La Sapienza, Roma, Itals

Sezione INFN di Ferrara, Ferrara, Italy

"Unversitá ai Modena e Reggio Emilia, Modena, Italy

Sezione IN di Cagllani, Caglari, Italy

${ }^{k}$ Universitiò di Roma Tor Vergata. Roma Italy

${ }^{\prime}$ CNAF-INFN, Bologna, Italy

"'Università di Ferrara, Ferrara, Ital,

${ }^{n}$ LAL, Université Paris-Sud, CNRSIN2P3, Orsay, France

${ }^{\circ}$ Università di Cagliari, Cagliari, Italy

Unversiáa al Koma La Sapienza, Roma, Ital 


\section{Dataset}

\section{- We took special "High Luminosities" runs in 2012 and 2018}

Calo measured luminosity 2012

$$
\begin{aligned}
& 4 \times 10^{32} \mathrm{~cm}^{-2} \mathrm{~s}^{-1} \\
& 5 \times 10^{32} \mathrm{~cm}^{-2} \mathrm{~s}^{-1} \\
& 6 \times 10^{32} \mathrm{~cm}^{-2} \mathrm{~s}^{-1} \\
& 8 \times 10^{32} \mathrm{~cm}^{-2} \mathrm{~s}^{-1} \\
& 10 \times 10^{32} \mathrm{~cm}^{-2} \mathrm{~s}^{-1}
\end{aligned}
$$

Estimated peak luminosity

$$
\begin{aligned}
& 5.7 \times 10^{32} \mathrm{~cm}^{-2} \mathrm{~s}^{-1} \\
& 7.1 \times 10^{32} \mathrm{~cm}^{-2} \mathrm{~s}^{-1} \\
& 8.6 \times 10^{32} \mathrm{~cm}^{-2} \mathrm{~s}^{-1} \\
& 11.4 \times 10^{32} \mathrm{~cm}^{-2} \mathrm{~s}^{-1} \\
& 14.3 \times 10^{32} \mathrm{~cm}^{-2} \mathrm{~s}^{-1}
\end{aligned}
$$

Calo measured luminosity 2018

$1 \times 10^{32} \mathrm{~cm}^{-2} \mathrm{~s}^{-1}$

$1.1 \times 10^{32} \mathrm{~cm}^{-2} \mathrm{~s}^{-1}$

$1.4 \times 10^{32} \mathrm{~cm}^{-2} \mathrm{~s}^{-1}$

$2.4 \times 10^{32} \mathrm{~cm}^{-2} \mathrm{~s}^{-1}$

$3.1 \times 10^{32} \mathrm{~cm}^{-2} \mathrm{~s}^{-1}$

$5.8 \times 10^{32} \mathrm{~cm}^{-2} \mathrm{~s}^{-1}$
Estimated peak luminosity

$6.7 \times 10^{32} \mathrm{~cm}^{-2} \mathrm{~s}^{-1}$

$7.2 \times 10^{32} \mathrm{~cm}^{-2} \mathrm{~s}^{-1}$

$9.4 \times 10^{32} \mathrm{~cm}^{-2} \mathrm{~s}^{-1}$

$16 \times 10^{32} \mathrm{~cm}^{-2} \mathrm{~s}^{-1}$

$21 \times 10^{32} \mathrm{~cm}^{-2} \mathrm{~s}^{-1}$

$39 \times 10^{32} \mathrm{~cm}^{-2} \mathrm{~s}^{-1}$ 


\section{Luminosity measurements from calorimeter}

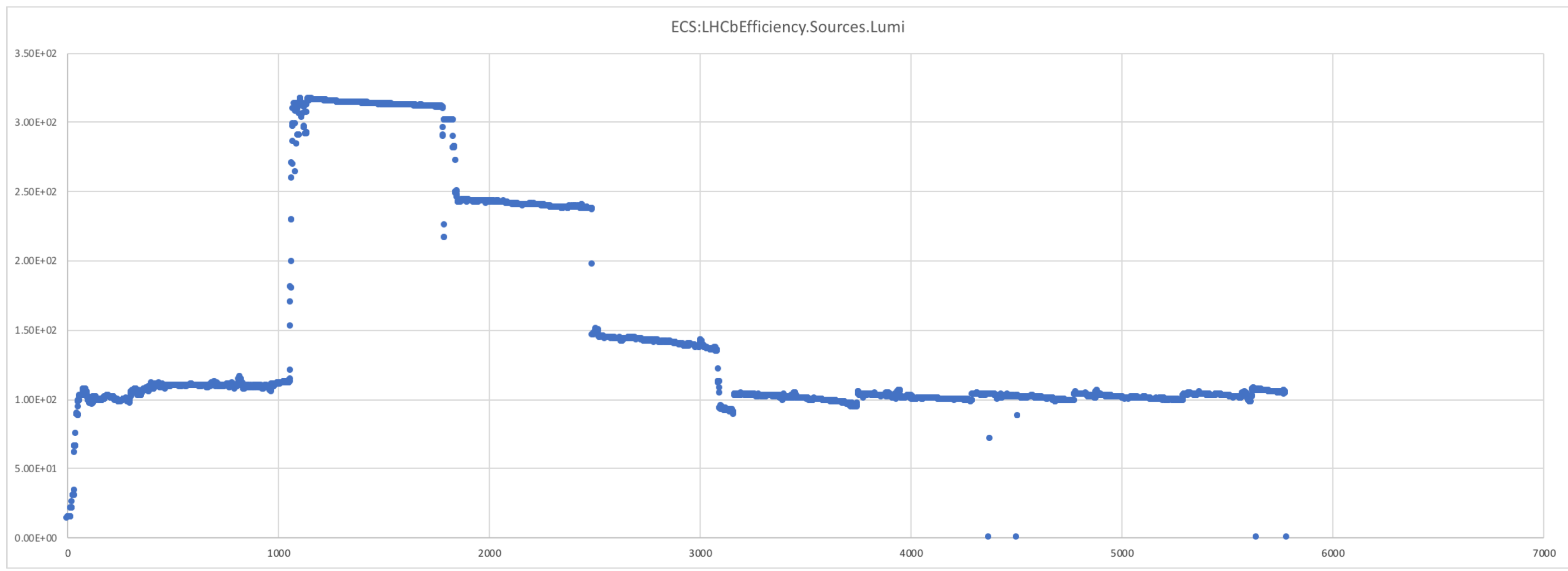

One of the fills of 2018 


\section{Rates distribution before $\&$ after deadtime correction}

In order to take correctly into account the dead time effect, it has to be corrected channel-by-channel (not in average);
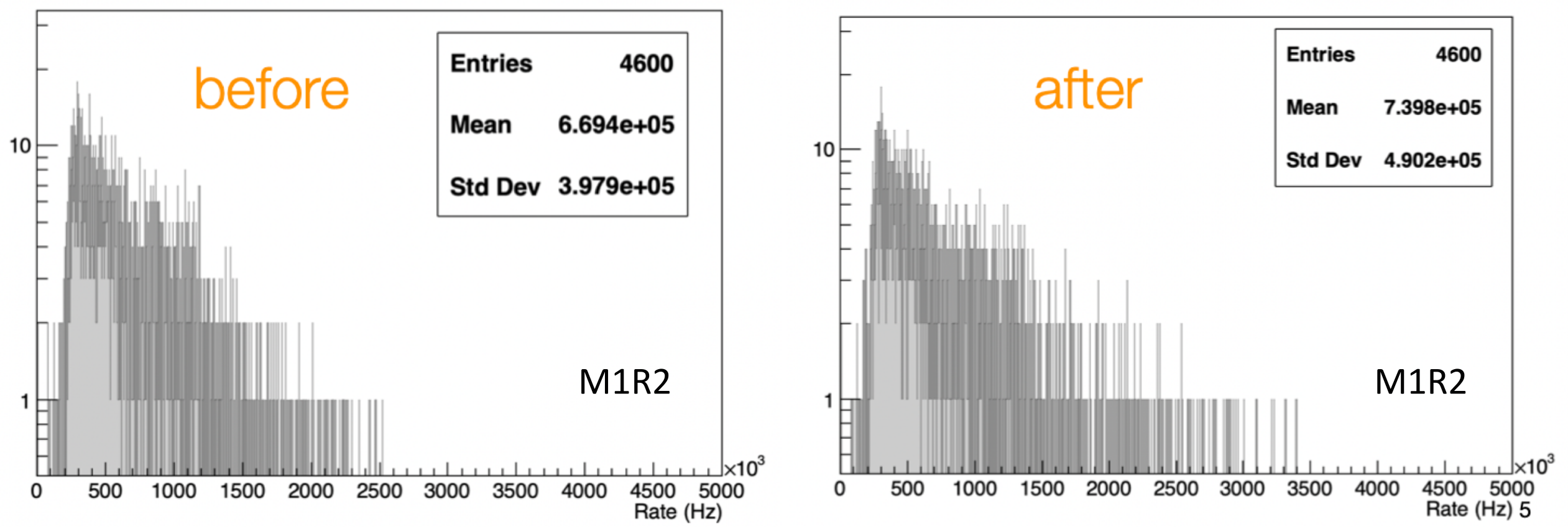


\section{LHCb luminosity: MUON vs CALO}

- The mean values of these distributions are plotted (before and after deadtime correction) w.r.t calorimeter measurements for M1 (2012)
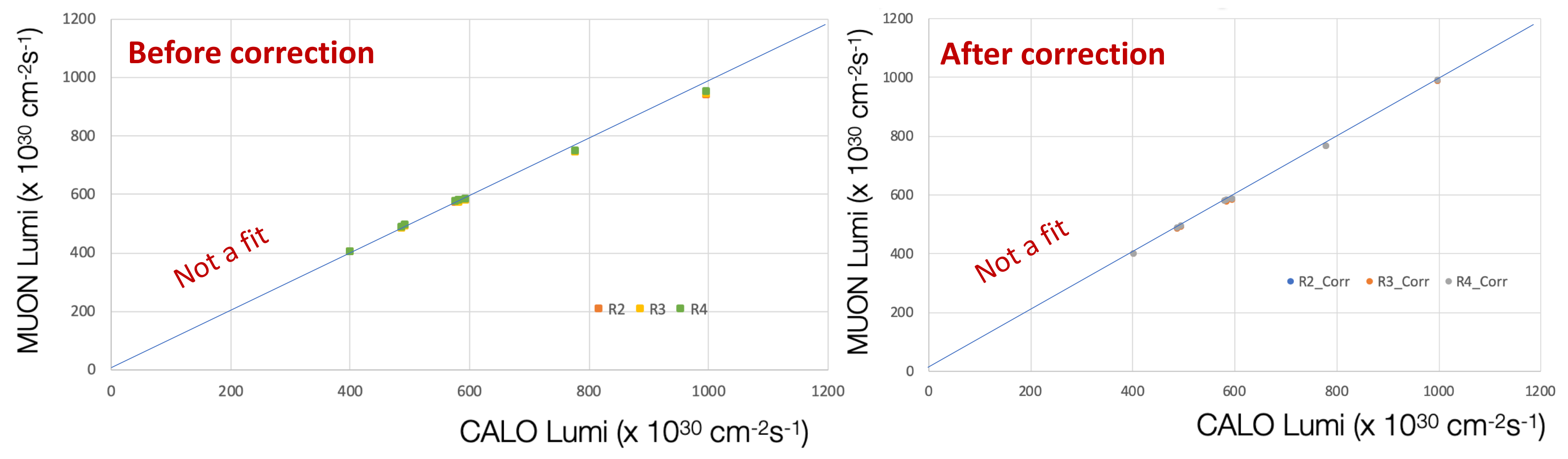


\section{The difference (\%) between the MUON and CALO measurements}

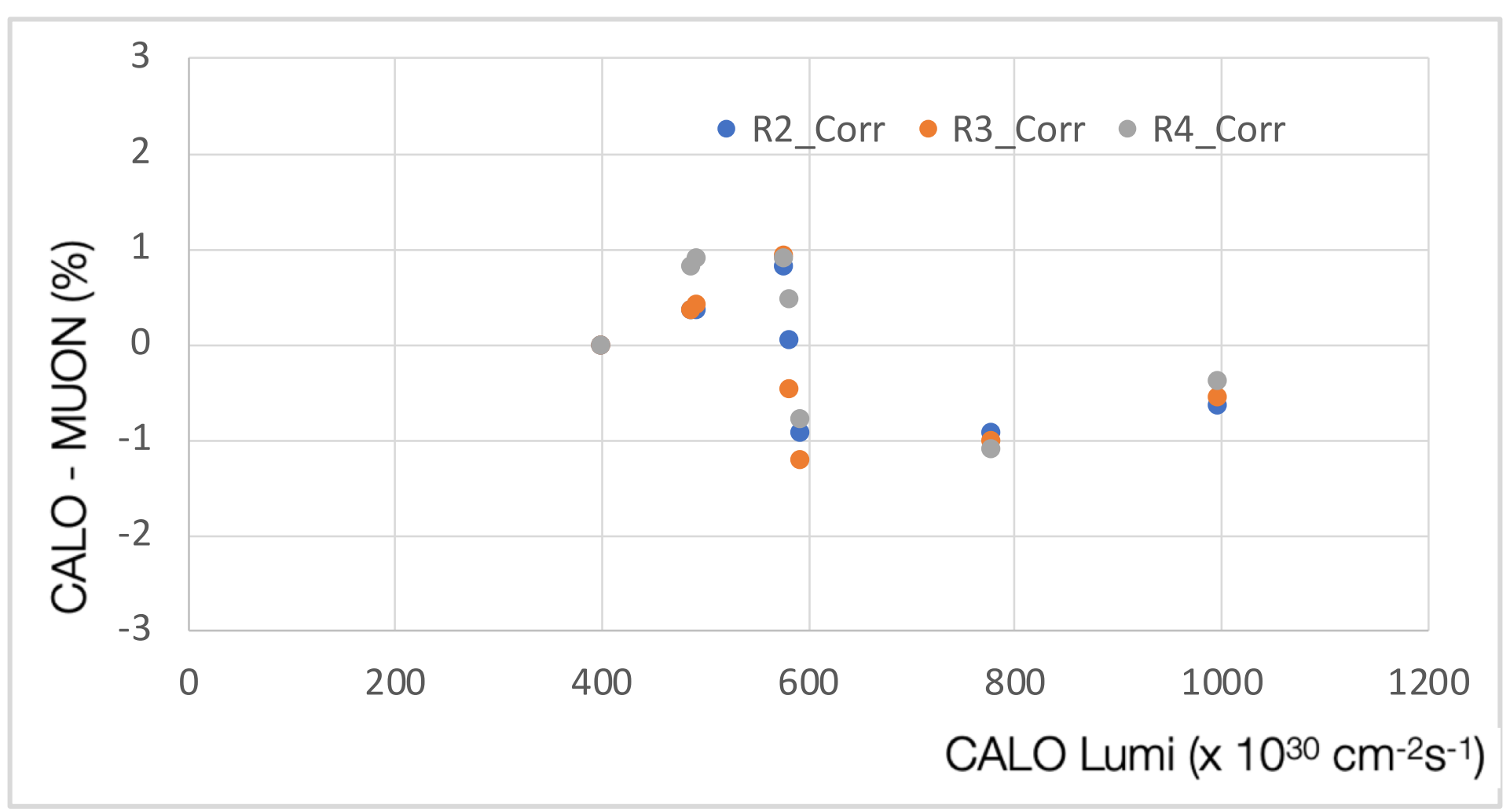

In the whole measured range, less than $1 \%$ difference 


\section{Self-consistency of the muon system}

histo_rapp_dtcorr_M2R3P1

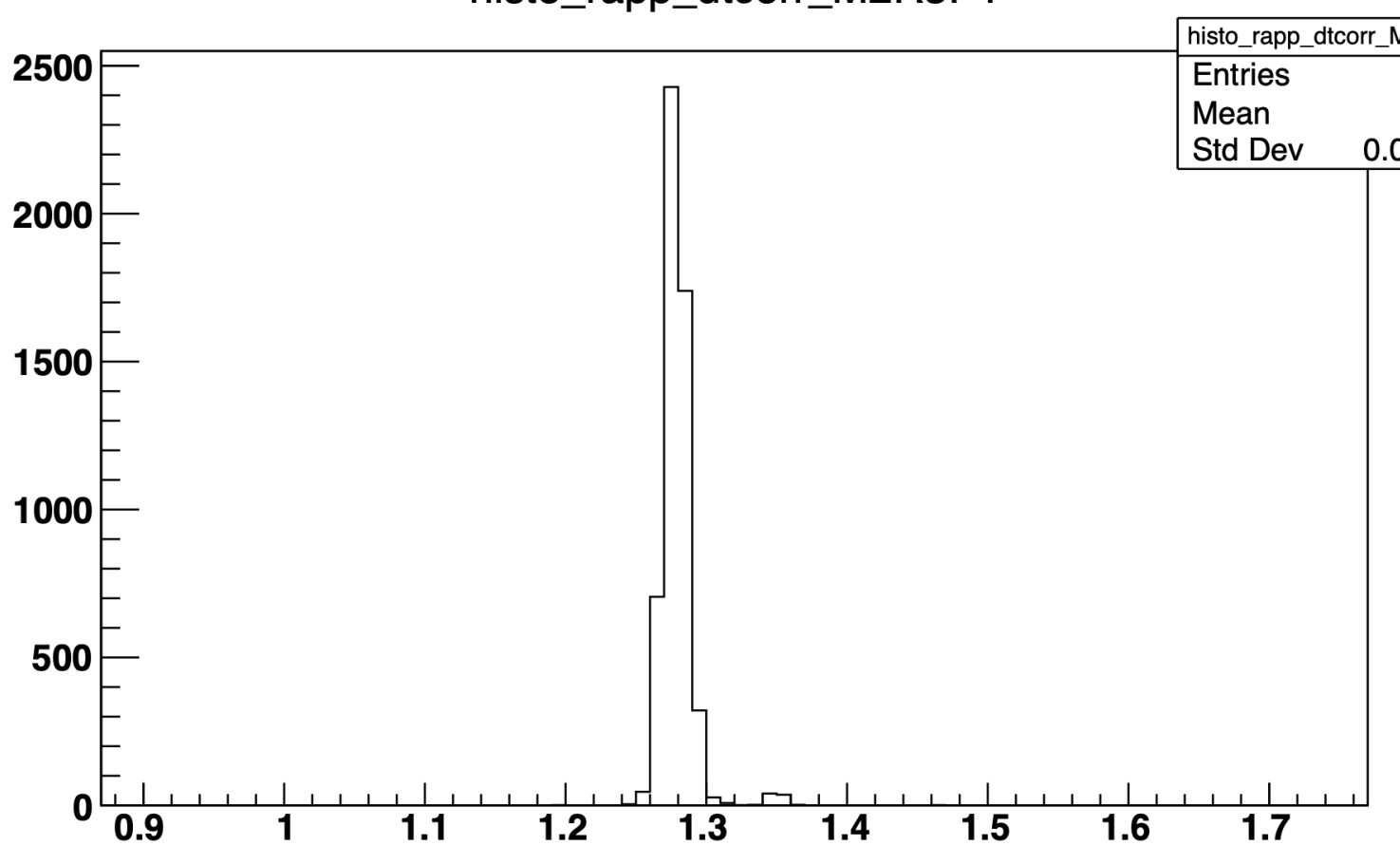

For each station and region the mean value of the channel by channel ratio of the rates taken at different luminosity for all regions.
$<-$ The method has a resolution of $1 \%$

histo_rapp_dtcorr_M2R3P1

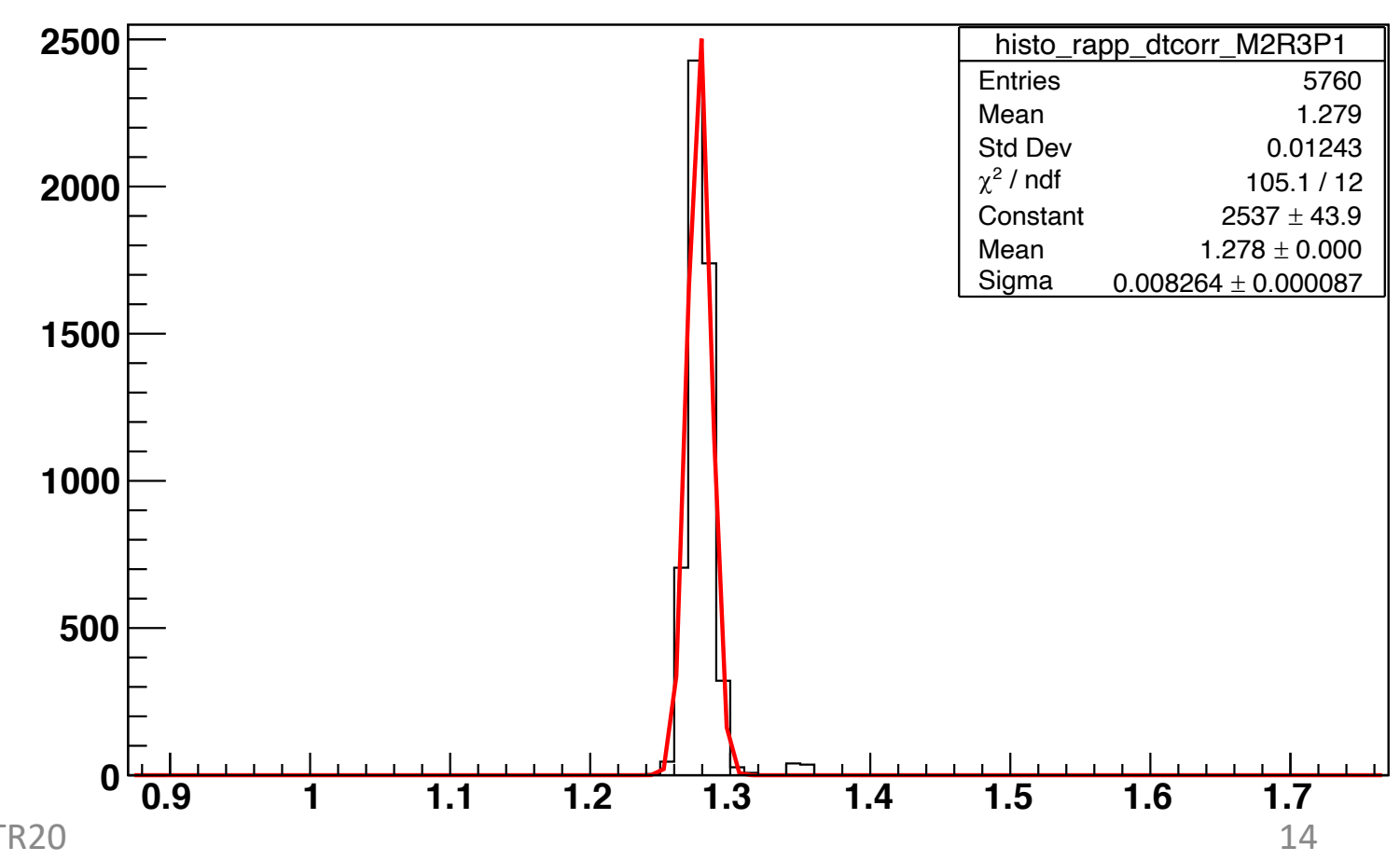




\section{Stability of the method in different regions}

Mean values of channel by channel rates ratio for lumi 140/100 (2018), weighted on CALO lumi

1.1

1.05

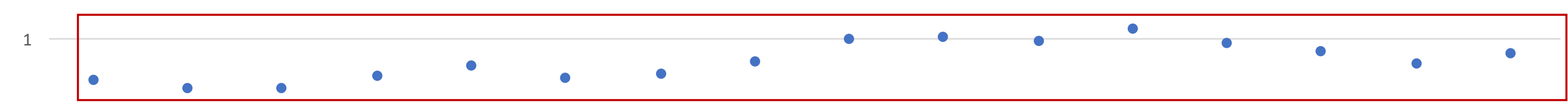

0.95

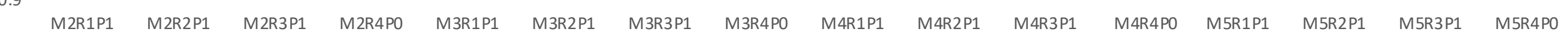




\section{Self-consistency of the muon system}
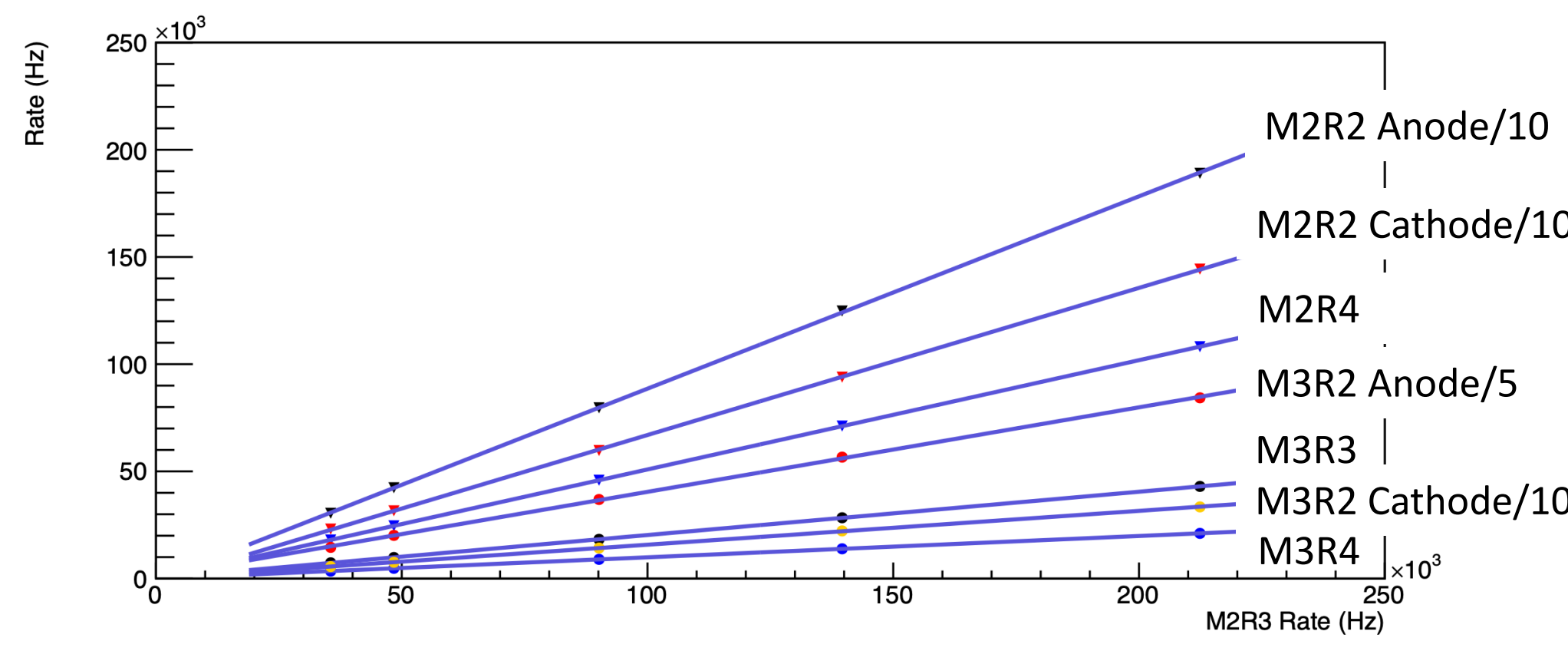

The distribution of the relative difference between absolute rates and the linear fit

Correlation between measured rates at the different luminosities

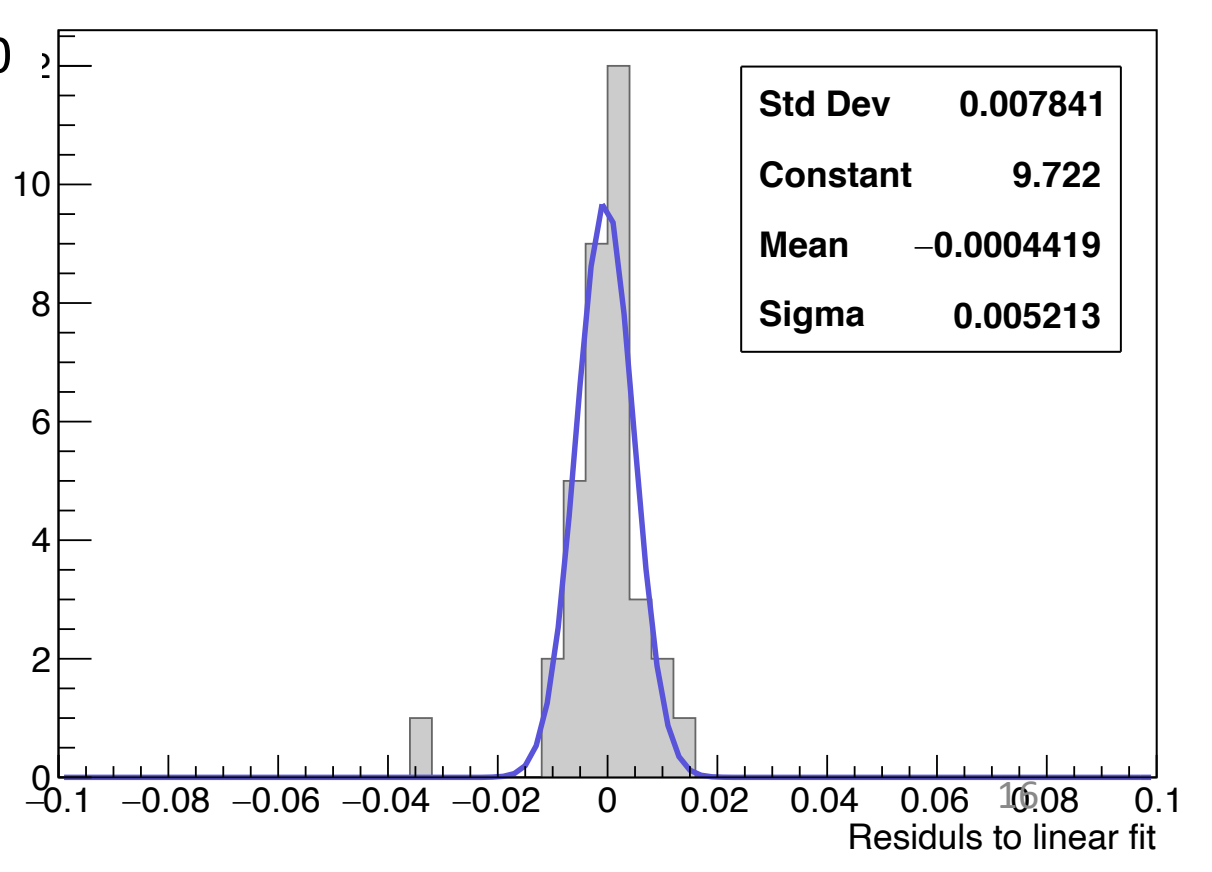




\section{High speed measurement with a few chambers}

- In order to have a fast version of the method, since the readout of the whole system takes $\sim 20$ minutes, we tried to analyze the data from only two chambers per region.

- Measured difference is around $1 \%$

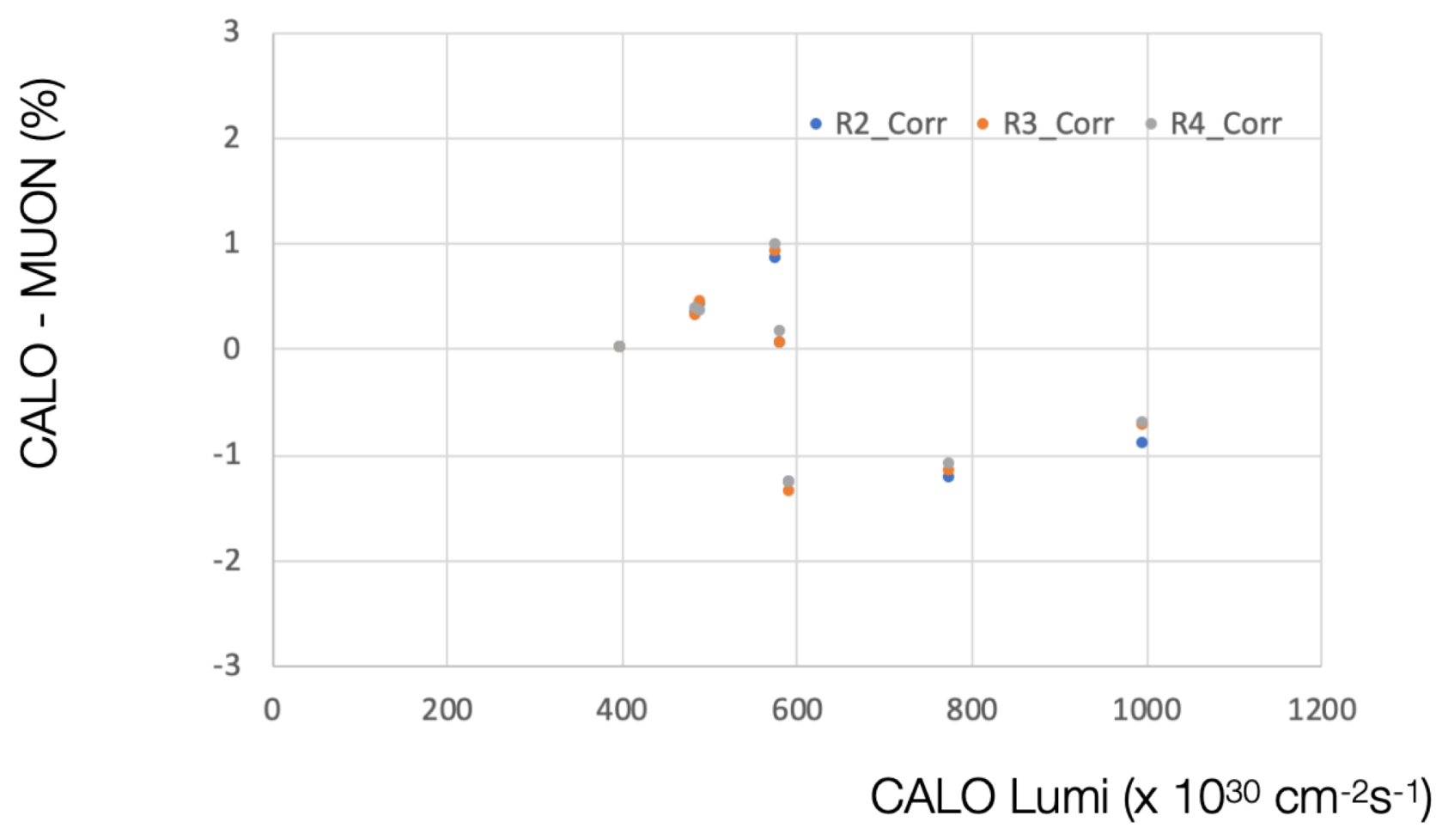




\section{Conclusion and next steps}

- In runs taken in 2012, the average rates in different regions (corrected for the dead time) are very good independent estimators of the $\mathrm{LHCb}$ Luminosity;

- The study of the 2018 data is ongoing

- The results are self-consistent within the whole muon system

- Even a few chambers can be used for these measurements

\section{Next steps:}

- Finish the analysis of 2018 data w.r.t CALO

- Perform a new scan in 2021 